\title{
LA EXPANSIÓN BIOGRÁFICA EN INVESTIGACIÓN EDUCATIVA. MOVIMIENTOS Y APERTURAS METODOLÓGICAS ${ }^{1}$
}

\author{
LUIS PORTA \\ https://orcid.org/0000-0002-5828-8743 \\ Universidad Nacional de Mar del Plata
}

RESUMEN En este artículo se comparten perspectivas y posturas sobre lo biográfico-narrativo gestadas a propósito de nuestras experiencias en el Grupo de Investigaciones en Educación y Estudios Culturales de la Universidad Nacional de Mar del Plata, Argentina. En los últimos veinte años, y al compás de movimientos específicos del campo pedagógico pero también de los marcos interpretantes a escala social, la investigación educativa nos ha conducido a ampliar nuestra concepción de la narrativa y, en paralelo, a diversificar las tecnologías y recursos metodológicos en los proyectos y programas académicos de los que participamos. El artículo narra las tramas de sentido que hoy configuran territorio y horizontes de lo educativo en el contexto de referencia, aludiendo a tres dimensiones que interactúan de modo solidario, y que pueden resumirse como los escenarios co-configurantes de nuestras prácticas, las diversificaciones temáticas y las alternativas metodológicas que mejor responden a estas mutaciones. Además de postular un análisis descriptivo de los efectos de la coyuntura para la investigación científica social, compartimos una serie de problemas para el campo de la educación y un abanico de opciones metodológicas que han emergido al abordarlas. Aludimos, en plena coherencia, a las implicancias subjetivas y las afectaciones recíprocas que estos movimientos académicos nos han provocado, en pos de asumir el compromiso pedagógico que da sentido a la investigación educativa.

Palabras clave: Campo pedagógico. Investigación educativa. Expansión biográfico-narrativa.

1 Este artículo se desarrolló en el marco de una estancia Posdoctoral en la Facultad de Filosofía y Letras de la Universidad de Buenos Aires, durante 2018 y 2019. Resolución 245/18. La misma se llevó a cabo en el equipo de trabajo que dirige el Dr. Daniel Suárez. 


\section{ABSTRACT BIOGRAPHICAL EXPANSION IN EDUCATIONAL}

\section{RESEARCH. MOVEMENTS AND METHODOLOGICAL OPENINGS}

This article presents several perspectives regarding the biographical-narrative domain which have been raised and nurtured by the work of the Research Team of Education and Cultural Studies at Mar del Plata State University in the last 17 years. The transformation of social scenarios and the pedagogic field that have coupled, in recent times, with the Team's efforts to approach central issues in education have resulted in an enlarged conception of narratives, the diversification of interests and the sophistication and expansion of tools for research. Thus, the text describes the three dimensions which compose the local, current academic landscapes and horizons-namely, the meaning-making social settings, the main contents and the wide array of resources and techniques that have been displayed in recent years-. It also addresses the subjective and personal affections that have been necessarily entangled with the academic work, both as a gesture of consistency and as a sign of genuine commitment to change in education, as well as in social life in general.

Keywords: Pedagogic Field. Educational Research. Biographical-narrative Expansion.

\section{EXPANSÃO BIOGRÁFICA NA PESQUISA EDUCACIONAL.} MOVIMENTOS E ABERTURAS METODOLÓGICAS

Este artigo compartilha perspectivas e posicionamentos sobre a biográfico-narrativa gestada e alimentada por nossas experiências no Grupo de Pesquisa em Educação e Estudos Culturais da Universidade Nacional de Mar del Plata, Argentina. Nos últimos vinte anos, e em sintonia com movimentos específicos do campo pedagógico, mas também com marcos interpretativos em escala social, a pesquisa educacional nos levou a ampliar nossa concepção de narrativa e, paralelamente, a diversificar tecnologias e recursos metodológicos. nos projetos e programas acadêmicos dos quais participamos. 0 artigo narra, precisamente, as teias de sentido que hoje configuram o território e os horizontes da educação no contexto de referência, aludindo a três dimensões que interagem solidariamente, e que se podem resumir como os cenários co-configuradores das nossas práticas, os diversificações temáticas e as alternativas metodológicas que meIhor respondem a essas mutações. Além de postular uma análise descritiva dos efeitos da conjuntura para a pesquisa científica social, compartilhamos uma série de problemas interessantes para o cam- 
po da educação e uma gama de opções metodológicas que surgiram ao abordá-los. Aludimos, com plena coerência, às implicações subjetivas e aos efeitos recíprocos que esses movimentos acadêmicos nos têm causado, para assumir o compromisso pedagógico que dá sentido à pesquisa educacional.

Palavras-chave: Campo pedagógico. Investigação educacional. Expansão biográfico-narrativa.

\section{Introducción}

Si bien la coyuntura actual se ha tornado propicia para los discursos apocalípticos, incluso impulsando en paralelo un conjunto de ingenuas ilusiones respecto del final de algunos males de la vida contemporánea, el transcurrir de la pandemia ha puesto de manifiesto la capacidad de resiliencia de la lógica colonial capitalística del mundo (ROLNIK, 2017). Frente a la obscenidad de las inequidades y las injusticias y el necesario deseo de gestar unas nuevas realidades circulan, no obstante, algunas prácticas sociales micro o meso subjetivas que reconocen una transformación en curso, aunque más discreta y silenciosa, y apostada en un largo plazo. La imagen de pachakutik, recuperada por Rivera Cusicanqui de la vertiente de su propia cosmogonía andina, logra capturar el tiempo de la convivencia de los violentos estertores de lo que resiste morir y las esperanzadoras promesas que inspiran la novedad suscitada por la creatividad constituyente de lo vivo y su diálogo diferido con las obstinaciones de algunas (otras) memorias.

Hacer investigación en este escenario, en el contexto de una universidad nacional argentina y en pos de comprender e intervenir pedagógicamente tanto los dominios más promisorios como los más estériles de la educación docente, exige una observación atenta de estos procesos de transmutación propios de la modernidad tardía. Pero también, específicamente, supone un reconocimiento de las afectaciones de la vida académica que se han suscitado en el enclave local al compás de las subversiones en las escenas más amplias de lo social. En este sentido, nos proponemos recuperar una memoria del Grupo de Investigaciones en Educación y Estudios Culturales que habitamos, no con el afán de cerrazón que supondría Una Historia, sino más bien con la vocación de reponer rostro y corazón a las trayectorias de la investigación educativa. Es precisamente en este colectivo, anudado por el interés en la educación docente en la universidad, donde se gesta y germina una importante expansión de lo biográfico que puede dar cuenta, en su singularidad, de los pulsos de lo académico al compás del movimiento social.

Con este propósito, comenzaremos describiendo las torsiones o convulsiones a nivel ontológico y epistemológico propias de la escena contemporánea. Deben comprenderse no solamente como un estado de situación, sino como el telar que nuestras propias prácticas de investigación han colaborado en hilar. Lo que se ha consolidado, solidariamente a propósito de las alianzas que las ciencias sociales han trabado con movimientos y otras agencias culturales, es una mirada o perspectiva no sólo sobre las posibilidades de producción de conocimiento sino, fundamentalmente, sobre sus fines o resonancias políticas.

En un segundo momento, daremos cuenta de los horizontes temáticos que los recorridos en investigación han ido inaugurando, bajo el signo de la turbulencia coyuntural pero ade- 
más de la mano de la promiscuidad cada vez más ineludible entre método y contenido. Las variaciones temáticas, así concebidas, pueden comprenderse como instancias en que los modos de hacer performan pedagogías, y estas, a su vez, hacen la investigación.

Finalmente, nos ocuparemos del estallido de recursos y herramientas metodológicas que, en los últimos 20 años, han tensado y enriquecido los proyectos pero, también, los sentidos y posibilidades para investigar. Este recorrido adjudica a la expansión biográfica sus colores y marca una tendencia hacia una aún mayor diversificación de los diseños y dispositivos para el trabajo de campo.

\section{Movimientos Epistémicos en la investigación²}

Si decidimos sostenernos, al menos hipotética o provisoriamente, en la composición revuelta de los tiempos que habitamos, y en esta condición que hace ya muchos años Sousa Santos describió como posición intermedia entre lo que muere y lo que soñamos/deseamos bajo el signo de la utopía (SOUSA SANTOS, 2003), la discusión respecto de lo epistemológico no puede desentenderse del arrastre que ha implicado para el plano de las ontologías. En esta misma línea Rolnik plantea que

[...] una atmósfera siniestra envuelve el planeta. El aire del ambiente, saturado de las partículas tóxicas del régimen colonial-capitalístico, nos sofoca. Con sucesivas transmutaciones, este régimen viene perdurando y sofisticándose [...] (ROLNIK, 2019, p. 25)

Es en virtud de esta percepción compartida que hablamos de epistemes, en un espíritu que linda con las reflexiones foucaultianas

2 Estas reflexiones son imposibles sin las presencias de los y las colegas del equipo de investigación. En este caso, especialmente María Marta Yedaide, Jonathan Aguirre y Francisco Ramallo a quiénes agradezco profundamente.
(FOUCAULT, 1995) pero se resiste a ser inscripto en unas cartografías enteras. En otras palabras, no tomaremos la episteme en un sentido completo, ni explícito ni subterráneo, que podría, por ejemplo, resonar en la frase "episteme moderna", sin negociar de antemano que es una estrategia analítica para agrupar, en una etiqueta, una yuxtaposición desprolija e inestable de una infinidad de sentidos sociales superpuestos. Guiados por la imagen poderosa de lo ch'ixi (RIVERA CUSICANQUI, 2016), las epistemes que se mueven pueden dar la impresión de síntesis cuando en realidad se trata de un efecto de la mirada, que uniformiza lo que se ha amontonado, superpuesto, de diversa naturaleza o composición, en la representación final.

También podriamos recurrir a la imagen que Cristina Martínez (2017) toma de Turillo (y este de una supuesta posible alusión a Gilles Deleuze) y que piensa la historia como una tela. Si estiramos la imagen y nos sumamos al experimento de representar la realidad, y no sólo la historia, como un paño, parece tener sentido pensar en una integralidad aparente que, además, dibuja figuras en sus movimientos. Esto nos habilita para exponer un segundo posicionamiento que pone el acento en las relaciones y ya no la ilusión de objetualidades preexistentes.

Sin lugar a dudas, estos puntos de vista que enunciamos, y que deben leerse como unas lentes interpretantes que contextualizan (configuran y componen solidariamente) el presente escrito, intiman con los despliegues del nuevo materialismo, los múltiples dominios que admiten agruparse (no sin cierta incomodidad) como cosmogonías indígenas, el posthumanismo y anti o post antropocentrismo, los feminismos y teorías de lo queer, el materialismo vitalista, las construcciones que han tomado por eje la enactuación y la performatividad, las antropologías y sociolo- 
gías del cuerpo y las emociones, las ontologías relacionales, etcétera. No desconocemos en esta enunciación una inclinación que pareciera emular al idioma analítico de John Wilkins, de José Luis Borges, tal y como lo trae a colación Foucault en Las palabras y las cosas (2011), que suponemos se agrava con el uso de la palabra "etcétera". Quienes patrullan celosamente las fronteras, sostienen y vigilan el apego a las buenas costumbres en la Academia, reforzando con sus dictámenes y juicios unos regímenes de poder particulares (DENZIN Y LINCOLN, 2011), seguramente se alarmen ante esta clasificación en apariencia bizarra e inconsistente. Que todas estas posturas o marcos interpretantes de la realidad y la ciencia convivan, a pesar de pertenecer originalmente a distintos campos de saber, o a porciones de ellos, o a tradiciones no doctas incluso, no constituye solamente un esfuerzo de compilación de LUPTON (2019), sino el reconocimiento de la importancia de registrar, como en un catálogo desordenado y "arrizomado", la escala y significancia del estallido vital de lo diverso y la necesaria promiscuidad que nuestras múltiples afiliaciones estimulan. Tal vez sea conveniente, en todo caso, pedir asilo en la caracterización de Suely Rolnik de estos tiempos convulsionados, en los cuales empezamos a advertir en el plano de existencia del saberde-lo-vivo la inadecuación de los territorios de ese segundo plano, más racional que también habitamos, y que podemos comprender como la superficie topológico-relacional del mundo (ROLNIK, 2017). De acuerdo con esta mirada, nuestra experiencia humana estaría desplegada en el continuo entre el mundo que hemos nombrado y "conocemos" y aquel otro dominio de la existencia al que accedemos a través de capacidades como la intuición. Esta imagen de doble superficie en un continuo explicaría la concurrencia, desde variados enclaves y circunstancias de las experiencias vitales, de ciertos trazos que buscan destituir nuestras representaciones del mundo en pos de hacer lugar para aquello que, fiel a los ritmos de todo lo que palpita, desea germinar. Habría en estos tiempos una crisis, entonces, entre nuestros modos de concebir-nos y aquello que adviene, y estaríamos entonando, desde territorios diferentes, el canto compartido orientado hacia la transformación de nuestras matrices semióticas.

No es sencillo, por supuesto, en este tránsito hacia horizontes aún inéditos, viajar sin las guías que han resultado hasta aquí fiables. La escala de la aventura exige el paulatino abandono de las certezas y la convivencia desordenada de despuntes en apariencia inconexos. De allí que el esfuerzo de Lupton constituya una excelente herramienta, tanto para otear los paisajes que se presentan como para ir familiarizándonos con la desestabilización de los fundamentos de la modernidad/colonialidad-aquellos cuya condición mitológica ha sido irremediablemente expuesta-.

En esta empresa de reseñar las reconfiguraciones paisajísticas que se van sucediendo en los territorios de la investigación, podríamos económicamente centrar el análisis en la fuerza y oportuna multiplicación de los "giros". Nos gusta jugar con este concepto de giro, toda vez que no necesariamente significa un movimiento circular, sino que también en la acción de girar la implicación de volver sobre uno mismo manifiesta la posibilidad de relectura, resignificación y viaje hacia el interior/exterior. La resonancia de los giros hermenéutico y lingüístico, a pesar del paso del tiempo, es todavía estridente. Tal vez su potencia no radique ya en las interpelaciones que oportunamente suscitaron, sino en la habilitación de los giros ontológico y narrativo, principalmente, y otras torsiones epistemológicas relacionadas, como el giro afectivo, el giro erótico y los otros tantos que se insinúan ya en el por-venir. 
En el territorio de la investigación en ciencia social, en general, y educativa en particular, la aparición de lo post cualitativo, las anti-metodologías, los enfoques performativos y aquellos basados en las artes, el sesgo autoetnográfico y las redefiniciones respecto de las actuaciones de los sujetos participantes de las experiencias de investigación tal vez representen los movimientos más recientes y radicales. Después de todo, "debemos aprender a ver más, oír más, a sentir más" (SONTAG, 1984, p.30).

La postura post cualitativa- cuyo referente más notable es sin dudas Elizabeth St. Pierre- es heredera de la inconformidad de lo postestructuralista y lo poscolonial, pero está también afectada profundamente por los desarrollos de la teoría cuántica y los aportes de Deleuze y Guattari. Interpela particularmente al Humanismo, la Representación, el Dato y al tratamiento de los Sujetos de la investigación (siendo las mayúsculas nuestras, en pos de enfatizar el carácter esencialista implicado), y denuncia especialmente la pretensión de hablar por los otrxs y construir-lxs (ST. PIERRE, 2013, 2014). Con apoyos en el nuevo materialismo, nuevo empirismo y el giro ontológico, dice independizarse de métodos o reglas- al punto de calificar como estéril o vano cualquier intento por enseñar o aprender a investigar- mientras se afecta, en cambio, por la condición enredada, emergente y habitual de la experiencia humana (ST. PIERRE, 2017). En intimidad con los marcos interpretantes que adopta, lo post cualitativo descree de la posibilidad de vacío teórico y expone la etnografía previa de larga duración; con estas declaraciones, inscribe los procesos de investigación en el continuum de la experiencia siempre significada, desmontando las ilusiones de separación tajante de los modelos epistemológicos de la modernidad/colonialidad. En términos concretos, admite con-fusiones entre lo vivo y lo muerto, el dato y el sujeto, lo material y lo inmaterial, lo cultural y lo natural, restituyendo la integralidad de las posiciones vitalistas. Esto conduce, tal como propone NORRDSTROM (2017), a optar por coreografias de territorializaciones y desterritorializaciones sucesivas y permanentes, que nos inscriben y exilian, alternativamente, de los modos más clásicos de performar la ciencia.

Pese a la fertilidad de esta mirada, que manifiesta gran sensibilidad a los gestos disruptivos de la escena contemporánea que describíamos al inicio, la postura post cualitativa no está exenta de críticas. Las más interesantes señalan la excesiva sofisticación de los argumentos y el carácter hiper encriptado del léxico comprometido en este enfoque. Estos modos de decir-se terminan por crear nuevas esencialidades a la vez que se enredan en la matriz binaria que intentan desarmar. Con sus piruetas intelectuales y sus apetitos por lo abstracto, terminan además funcionando como mecanismos de exclusión, que exacerban las distancias y las jerarquías con altos costos sociales y políticos (GERRARD, RUDOLPH y SRIPRAKASH, 2017).

Parece justo señalar que lo post cualitativo, tal vez en sus momentos territorializantes, coincide en gran medida con las formas más radicales que asume la investigación bajo ese otro más antiguo pero suficientemente amplio paraguas de la Investigación Cualitativa (DENZIN y LINCOLN, 2011). Las contribuciones publicadas en Sage durante los últimos 20 años dan cuenta de grados de radicalidad y diversificación dentro de este dominio que hacen casi inverosímil que agrupe al pospositivismo con posturas totalmente desancladas de las tecnologías tradicionales de la ciencia. En esos bordes podemos situar a las investigaciones basadas en las artes (FINLEY, 2015), lo autoetnográfico (HOLMAN JONES, 2015), lo performativo (DENZIN, 2019) y los travestismos 
hacia el otrora modelo particular de la investigación acción participativa (KEMMIS y MCTAGGART, 2013). En términos generales, se trata de la recuperación de esferas de la vida común y, a la vez, resistencias políticas a los regímenes de poder moderno/coloniales de la ciencia. Las artes y lo poético no sólo desafian la literalidad como modo predilecto de sostener la confianza en la Verdad Revelada mediante la Razón Humana; al hacerlo, cuestionan el cálculo -ese modo tan particular de este tiempo-espacio histórico que violenta lo vital para ajustarlo al concepto (CANDELERO, 2016) - . Por supuesto, en este mismo movimiento se subvierte el mito de la objetividad, al punto de preferir la abierta manifestación de las marcas ideológicas y biográficas de los investigadores. Esto se manifiesta tanto las investigaciones interesadas en las artes (aquellas que no toman lo artístico como hecho a traducir, sino como modo de interpelar y producir conocimiento y saber común y necesario) como lo autoetnográfico, que además reintroduce el valor de la historia singular (como único relato finalmente posible pero también como posibilidad de lectura del diálogo entre lo propio y lo común). Estas formas de practicar la investigación comprometen una radicalidad inusitada, todavía fuertemente resistida por los mecanismos de control en vigencia. Paulatinamente, no obstante, van tomando fuerza no tanto gracias a la flexibilización de los regímenes de gestión de las instituciones académicas, sino más bien por su importancia y valor político y social, de espaldas, incluso, a estas agencias históricamente monopolizadoras de la producción de conocimientos legítimos (LINCOLN, 2011). La performatividad, por ejemplo, que hace pie en la teoría feminista y su ampliación de la original premisa austiniana, invita a componer, enactuar, reescribir, recomponer y revivir el texto social a partir del teatro foro (DENZIN, 2019). Allí se abandona sin pudor la pretensión universalista, mientras se estrechan maravillosamente los procesos y productos de investigación a las condiciones vitales y los sujetos implicados. Es por esto que, sostenemos, asistimos a una paulatina generalización de lo que alguna vez fuera el enfoque de la investigación-acción- participativa, a medida que las necesidades de los sujetos (SEGATO, 2015), el reconocimiento de las implicaciones y las urgencias de las gentes van trans-vistiendo, saludablemente, a la investigación en una práctica comunitaria, asumiendo un sesgo hoy ineludible para pensar la práctica académica honesta y políticamente responsable.

\section{Aperturas Temáticas: mutaciones afectadas por los tiempos y sus tecnologías}

Podríamos cartografiar los movimientos de la comunidad investigativa que integramos y nos nuclea en clave de mutaciones. La metáfora no es inocente, puesto que muchas de ellas se invisten, precisamente, de la perspectiva vitalista mencionada en el apartado anterior. Al componer una historia-memoria particular y específica, la primera mutación temática implicó el abandono de cuestiones propias del campo de la didáctica del nivel superior, e inscriptas en su agenda (LITWIN, 1996), hacia una preocupación por las narrativas biográficas de los docentes. El paso del interés por la buena enseñanza a los profesores memorables (ÁLVAREZ, PORTA, SARASA, 2010) implicó adentrarse en un dominio de exclusiones y resistencias y a cuestionar, tímidamente en un principio pero con gran decisión y coraje luego, los sentidos prevalentes y las demarcaciones que el campo pedagógico establecía para el abordaje de las emociones y los afectos (PORTA, ÁLVAREZ y YEDAIDE, 2014). Podríamos decir que, en esta dirección, se produjo un segundo desplazamiento, del interés por las configuraciones de vidas 
de grandes docentes hacia el terreno más abstracto pero también más situado de las autorizaciones discursivas. Los aportes de los estudios culturales, que habían acompañado los primeros desarrollos prácticamente en paralelo, se revitalizaron a propósito del pensamiento descolonial y las alteraciones que suscitaba sobre la base de pensar que colonización y colonialidad aludían a dos dimensiones imbricadas pero diferentes de las experiencias de los pueblos subalternizados. $Y$ es de la mano de los rastreos de las propias marcas coloniales que la lente vuelve a mirar y mirar-nos, allí donde el territorio había sido negado (signado por unas historias y unas marcas locales propias) y los cuerpos invisibilizados.

También a partir del interés por las biografías docentes se gestan otras tres mutaciones concomitantes. Podríamos caracterizar el primer gran desplazamiento desde un sentido didáctico/disciplinar hacia una preocupación más pedagógica/política (YEDAIDE, 2016). Las contribuciones de los pedagogos críticos, especialmente la obstinación de Peter McLaren con las disputas semióticas en el campo de lo cultural, así como la insistencia de Michael Apple de mezclar academia y activismo, estimularon una línea de desarrollo que ha intentado desde entonces dibujar nuevos mapas de condiciones y condicionantes, atmósferas vitales, que resucitan las urgencias políticas del campo. Estos aportes, en combinación más o menos problemática con las teorías descoloniales, han conducido a la exploración de los modos en que la modernidad/colonialidad garantiza su pervivencia en los procesos de educación docente e investigación educativa, mientras se gestan-en el mismo movimientomúltiples formas de resistencia y reexistencia (PORTA y YEDAIDE, 2017).

Otro importante desplazamiento en los "objetos" de interés se materializó, en simultaneidad, a propósito del método biográfico narrativo en sí mismo. Podríamos decir que hemos ampliado la mirada, desde un inicial abordaje más instrumental hacia el ensanchamiento de la dimensión metodológica en términos de posicionamiento ético-onto-epistémico (KUBY y CHRIST, 2018). El método, en diálogo con los giros a los que hicimos referencia anteriormente, desbordó su función inicial como herramienta de gran potencia heurística para conducirnos a debates inherentes a la condición narrativa de la experiencia humana. La narrativa, en sí, se ha transformado en una cuestión interesante, y con ella los modos en que devenimos, construimos y somos construidos en la trama de la vida social. Y, en intimidad con estas inquietudes, la disputa de la Historia, la Razón, el Sujeto, así como otros Grandes Relatos de la modernidad/colonialidad, nos han hecho virar hacia la gestación de contra-memorias y la reinterpretación de las políticas en clave de los rostros y las historias personales en que se encarnan (RAMALLO, 2017; AGUIRRE, 2019).

Un tercer desplazamiento concomitante respecto de los temas de nuestro interés se anudó a las emociones, los sentimientos y la construcción social de lo abyecto. Bajo la influencia de los feminismos radicales y las teorías y pedagogías cuir, la recuperación o reposición de la sensibilidad ha supuesto desvíos, errancias y merodeos alrededor de ciertas ficciones modernas y, especialmente, de sus artificios. Por un lado, importan la subalternidad y su permanente reedición, pero también las políticas de afiliación y afirmación de las comunidades despreciadas (WAYAR, 2018; FLORES, 2017; SEDGWICK, 2018; HALBERSTAM, 2016). Por otro, se despliega la promiscuidad, extendiendo la deserotización de la experiencia sexual a un patrón higienizante que aliena, extranjeriza y promueve la necrofilia en todos los ámbitos de la vida, incluidos los hábitos epistemológicos (HARAWAY, 2016). Estas costumbres moder- 
nas son desafiadas, también, desde las otras ciencias, que postulan al compás de la física cuántica y las teorías de los sistemas complejos una biología pensada desde la co-evolución, la otredad significativa y la naturocultura (HARAWAY, 2015).

También en el seno de este tercer desplazamiento nació la vocación por explorar lo íntimo y lo éxtimo, la belleza, el fracaso, el arte, la performance, los cuerpos, los deseos, la erotización, la creatividad, la imaginación y el goce. Vueltos hacia lo educativo en nuestras coyunturas, todos estos dominios de interés han exigido un compromiso ético, de las primeras personas del plural y del singular, además de las responsabilidades políticas inherentes a las prácticas de producción de conocimientos en la universidad.

Podríamos decir que la profundización en el campo de la didáctica del nivel superior $y$, particularmente, las prácticas docentes (BRANDA, 2017; MARTÍNEZ, 2017) ha continuado la vocación inicial, ineludiblemente afectada no obstante por las interpelaciones epistemológicas y las pedagogías resultantes. La construcción de la identidad docente, en esta misma línea, ha sido abordada narrativamente, dando lugar a interpretaciones fecundas que han asumido, paulatinamente, formas de decir-se desafiantes y osadas para la socialización de las investigaciones (DE LAURENTIS, 2019 SARASA, 2016). Se han ampliado y complejizado, asimismo, dimensiones de la práctica docente lindantes con la ética, el humor y las condiciones de hospitalidad de la enseñanza (FLORES, 2018), así como contenidos interesados por los cuerpos, las sensibilidades y las estéticas (LAZZARIS, 2020; TRUEBA, 2019)

Todas estas líneas temáticas, que podrían haberse representado en un principio como un ramillete enlazado por esa intención primigenia del Grupo de comprender los procesos de buena enseñanza en la formación docen- te en la universidad, se muestran ahora más justamente representadas como un rizoma. La diversificación de los intereses y la multiplicación exponencial de campos de saber fronterizos que se van entramando en las preocupaciones contemporáneas han conducido a la gestación de varios proyectos y grupos de investigación que generan, a su vez, esferas de atracción e influencia dentro del campo académico pero también en los inter-territorios con los que comparten intrigas.

Finalmente, tal vez como conclusión paradojal, es la común afiliación a lo biográfico-narrativo lo que sigue enhebrando lo distinto bajo un signo común.

\section{Diversidad Metodológica}

La perspectiva biográfica-narrativa, como mencionamos en líneas anteriores, se fue entramando, en nuestra comunidad de investigadores, desde un doble sentido: como método o metodología de investigación y como relato en la acción que propugna el sentido de una vida puesta en contexto y en el mundo (PORTA, AGUIRRE y RAMALLO, 2018). Inicialmente, decíamos, nos permitió poner a la docencia universitaria en términos vitales con la investigación sobre las prácticas de enseñanza; luego y producto de las indagaciones doctorales de los miembros del equipo, el enfoque posibilitó expandir esos sentidos y significados hacia nuevos interrogantes de investigación produciendo desplazamientos temáticos y metodológicos que enriquecieron el trabajo. Esta fertilidad de la que hemos dado cuenta es precisamente lo que nos anima a sostener la potencia expansiva que tiene la perspectiva biográfica y narrativa en la recuperación de los sentidos que los sujetos le otorgan a sus cotidianos vitales y educativos.

Nos gustaría ahora virar hacia los soportes metodológicos que los movimientos epistémi- 
cos y las aperturas temáticas que fuimos habitando en los últimos años de producción del GIEEC requirieron, para su despliegue. A modo de cartografía metodológica e instrumental, y recuperando recursivamente lo compartido en los apartados anteriores, podríamos pensar los recorridos de investigación del GIEEC en cuatro momentos o etapas, en las cuales lo biográfico-narrativo fue asumiendo, en el devenir de las búsquedas e indagaciones, diversos matices epistémico-metodológicos que abonaron sus producciones y las de cada uno de sus miembros.

Tal como adelantáramos, los primeros proyectos del grupo, desarrollados entre los años 2003 y 2007, tuvieron como principal objetivo indagar las buenas prácticas de enseñanza que se suscitaban en la formación del profesorado universitario de la Facultad de Humanidades de la Universidad Nacional de Mar del Plata. Aquí, formación docente, enseñanza y didáctica fueron los núcleos que vertebraron las investigaciones, los cuales se cimentaban en los principales rasgos de lo que se denominó la "Nueva agenda de la didáctica" (LITWIN, 1996) hacia finales de la década de los 90.

A los efectos de identificar a aquellos docentes considerados ejemplos de buena enseñanza ${ }^{3}$ el soporte metodológico utilizado fue la administración de encuestas semi-estructuradas a estudiantes avanzados de diferentes carreras de los profesorados. En nuestro caso, las encuestas fueron diseñadas con un formato semi-estructurado, lo cual permitió que el participante encuestado pudiera brindar no solo elementos de corte estadístico, sino rasgos cualitativos sobre la enseñanza de los

3 El concepto ha sido revisado y puesto en cuestión a partir de la segunda década del siglo XX por estudios que se interrogaban su conceptualización modélica y recuperando matices epistemológicos descoloniales y críticos sobre la noción binaria y moralizante entre lo bueno o lo malo (YEDAIDE, 2016). Asimismo la cuestión valorativa también se ha puesto en cuestión producto de la reflexión en torno al origen mismo de dicha construcción de valores. (MARTíNEZ, 2017) profesores seleccionados e identificados como memorables.

Otro soporte central en esta primera etapa, como ha sido a lo largo de los más de 15 años de trayectoria del GIEEC, fue la realización de entrevistas en profundidad a los profesores memorables consignados como tales por sus estudiantes. Siendo coherentes con nuestros marcos conceptuales y metodológicos, aún hoy asumimos la entrevista como el principal instrumento de interacción en investigaciones cualitativas en ciencias sociales (GUBER, 2016; DENZIN y LINCOLN, 2015; TAYLOR y BOGDAN, 2007;). El guion oportunamente diseñado permitía a los entrevistados narrar sus prácticas, compartir sus concepciones respecto a la disciplina, a la enseñanza, a su vínculo con los estudiantes, y centralmente, aludir a sus experiencias familiares, educativas y formativas que, resignificadas en el relato oral, tenían una ligazón íntima con sus propias prácticas docentes en el momento.

En esta primera fase de trabajo las entrevistas posibilitaron comprender aspectos de la práctica docente no desde la prescriptividad del currículum formal, sino desde los significados que los sujetos le otorgaban en sus propias narrativas biográficas. Es por ello que en la segunda etapa de producción del GIEEC entre los años 2008 y 2013, el centro del análisis y de las indagaciones fueron las vidas mismas de los profesores memorables reconocidos anteriormente.

En dicha etapa, a diferencia de la anterior, las entrevistas recorrieron la vida personal y profesional de cada uno de los profesores, como así también sus propias concepciones sobre la enseñanza y la formación de docentes en términos del carácter social, histórico y territorial de la vida de los sujetos. En este sentido, el lugar de la experiencia es el registro sobre el que se profundizó y nos permitió, a partir de dichos relatos (auto)biográficos, la 
emergencia de categorías significantes para conceptualizar la docencia (PORTA Y AGUIRRE, 2020). En las entrevistas de esta etapa, lo personal y lo profesional se fusionaron en una amalgama vital que otorgó tanto a los docentes participantes como a los propios investigadores la oportunidad de aproximarse al cotidiano biográfico que configura gran parte de los aconteceres en el aula y en las prácticas educativas.

La tercera etapa de investigaciones del Grupo, que se concentra entre los años 2014 y 2016, atestigua la primera expansión propiamente dicha de la perspectiva biográfica y narrativa del equipo en términos metodológicos. El GIEEC albergó en este tercer momento cuatro proyectos de investigación que comenzaron a expandir los sentidos y significados del trabajo realizado en la primera década del equipo. Uno de ellos se orientó hacia el abordaje narrativo de la biografía escolar en las prácticas profesionales de estudiantes residentes del Profesorado de inglés (BRANDA, 2017). Otro posó la mirada en las potencialidades de la indagación narrativa en la educación superior a partir de relatos identitarios de estudiantes de la carrera del Profesorado en inglés de la misma Facultad (SARASA, 2016). Además, en un tercer proyecto, se habilitó la indagación de recorridos biográficos en instituciones de educación alternativa de la ciudad de Mar del Plata, Argentina. Finalmente la cuarta línea mantuvo el fundante abordaje biográfico-narrativo de los profesores memorables, en sus vidas, sus pasiones y sus prácticas en las aulas de la universidad (PORTA, AGUIRRE y RAMALLO, 2018).

En este sentido, los instrumentos que sirvieron de soporte metodológico para el despliegue de las investigaciones también evidenciaron su primera expansión. Esta etapa contiene una variopinta cantidad de instrumentos que visibilizan una polifonía a partir de la cual cada proyecto pudo indagar biográfica y na- rrativamente sus propios objetos. Entrevistas flash (DENZIN y LINCOLN, 2015), entrevistas en profundidad a docentes, estudiantes y adscriptos, grupos focales a profesores y graduados, etnografia de aula (ROCKWELL, 2009), diarios de campo (GUBER, 2016; TAYLOR y BOGDAN, 2007), documentos narrativos (PORTA, 2018), etnografía de documentos y materiales didácticos (ROCKWELL, 2009) fueron algunos de los principales soportes metodológicos que permitieron la expansión del enfoque y lo materializaron en la práctica investigativa concreta.

La actual etapa de investigaciones del Grupo ha evidenciado una mayor expansión en lo que respecta a temas e instrumentos de recolección de datos. A partir de diversos trabajos doctorales de los miembros del equipo, fuimos transitando desde el año 2014 algunos replanteos temáticos y particulares expansiones metodológicas del enfoque (auto)biográfico-narrativo en el campo de la educación superior argentina.

Una de las expansiones primigenias del GIEEC estuvo orientada a un campo de investigación abordado en extenso por producciones más clásicas, como es el caso de la historia de las prácticas educativas. Ramallo (2017) realiza un análisis histórico de "otras enseñanzas" en los Colegios Nacionales a mediados del siglo $\mathrm{XX}$, recuperando dichas prácticas desde el enfoque biográfico-narrativo con un marcado contenido que rompe la matriz clásica y académica con que ha sido caracterizada la enseñanza en dichas instituciones educativas. Allí se recuperan una serie de aspectos a partir de las voces de los protagonistas entrevistados y de diferentes materiales de archivos escolares indagados: "Desde el enfoque biográfico-narrativo discutimos y revisamos las miradas nostálgicas y desprovistas de la capacidad de acción de los sujetos, que suelen dolorosamente caracterizar al campo de la historia de la educación en general y los relatos sobre 
esta formación en particular" (PORTA, AGUIRRE, y RAMALLO, 2018, p. 174). Las entrevistas biográficas a graduados del Colegio Nacional, los documentos escolares que yacían en los archivos, los múltiples registros textualizados de la institución y la propia voz del investigador permitieron interpretar las prácticas de enseñanza en el bachillerato argentino desde diversos relatos que pusieron en jaque las visiones clásicas de la historiografía tradicional argentina.

La segunda expansión protagonizada a partir de las tesis doctorales está relacionada al campo de las políticas de formación docente y a las incipientes indagaciones sobre la profesión académica en las universidades argentinas. A partir de la investigación doctoral "La formación docente en Argentina. Un estudio interpretativo de las políticas nacionales. El caso de los Polos de Desarrollo" (AGUIRRE, 2019) el enfoque narrativo hace sus primeras incursiones en el área temática de las políticas educativas. Con el objetivo de interpretar los múltiples niveles de traducción del proyecto Polos de Desarrollo en la formación docente de nuestro país y las potencialidades y tensiones que éste asumió en su puesta en acto en el territorio institucional, se realizaron más de 40 entrevistas a gestores nacionales, coordinadores jurisdiccionales, referentes académicos, autoridades de los institutos de formación docente implicados, coordinadores locales, docentes y estudiantes. A partir de este registro polifónico de la política se van entramando las voces de los protagonistas, los documentos oficiales y el propio registro autoetnográfico del investigador, hilvanando una meta-narrativa interpretada desde sus problemáticas, sus tensiones y sus aportes al mejoramiento de la formación docente y sus instituciones educativas. Los hallazgos fueron presentados así como una narrativa biográfica de la política en sus diversos niveles. BOLÍVAR, DOMINGO y FERNÁNDEZ celebran esta manera de evidenciar los hallazgos puesto que "una buena investigación narrativa, [...] no es solo aquella que recoge bien las distintas voces sobre el terreno, o las interpreta, sino también aquella que da lugar a una buena historia narrada, que es en el fondo, el informe de investigación" (2001, p .207).

En la etapa que recorre desde el 2017 al 2020, el equipo consolidó sus investigaciones en torno a la configuración de identidades docentes (DE LAURENTIS, 2019). El enfoque metodológico narrativo permitió comprender la construcción identitaria de 27 colegas del ciclo de formación de profesores de la Facultad de Humanidades de nuestra universidad. Al ser la identidad un objeto de estudio por demás complejo y dinámico, se recurrió no sólo a las voces y narrativas de los participantes de la investigación, sino que se apeló a imágenes, dibujos, canciones, recuerdos personales, y algo aún más novedoso en nuestra comunidad de trabajo, se incorporaron metáforas en los registros (auto)biográficos de los profesores (LAKOFF y JOHNSON, 1995). Ese mosaico de instrumentos de recolección de datos brindó la posibilidad de interpretar las identidades docentes desde coordenadas cualitativas innovadoras.

Una cuestión que abonó significativamente la expansión instrumental del enfoque (auto) biográfico-narrativo en nuestras investigaciones fue la incorporación del diario autoetnográfico en las producciones doctorales del equipo (AGUIRRE, DE LAURENTIS y BOXER, 2019). Dichos registros narrativos, que el investigador completa desde el inicio de su actividad hasta su culminación, evidencian no sólo lo que va sintiendo el sujeto que investiga en el correr de tarea investigativa, sino sus propios aprendizajes y desaprendizajes en la medida que le va dando forma al trabajo de indagación. Este 
diario, luego, es incorporado a las tesis en diversos espacios según la dinámica que asuma cada escrito. En algunos casos, se incorpora como capítulo final al estilo de metacognición del proceso, en otros se suma al capítulo metodológico y, en ocasiones se despliega como apartados finales de cada capítulo escrito.

Para finalizar esta cartografía metodológica, debemos recuperar la más reciente expansión del enfoque. La misma versa en torno a las prácticas de enseñanza y sus vínculos con la corporeidad en los profesores memorables. Si bien las conceptualizaciones sobre la enseñanza y la memorabilidad de los docentes ya han sido abordadas por investigaciones del grupo, la incorporación de la dimensión corporal al análisis es absolutamente novedosa. A partir de una tesis doctoral recientemente presentada 4 , desde una perspectiva hermenéutica-interpretativa, nos propusimos comprender los sentidos a través de los que se expresa la corporeidad y la buena enseñanza en una de las profesoras memorables seleccionadas por sus estudiantes y que conforma el universo de 6 docentes con los que venimos trabajando desde el año 2008 (LAZZARIS, 2020).

En dicha indagación se aborda la problemática corporal a través de la mirada filosófica, en particular la fenomenología y distintas disciplinas que componen una epistemología convergente, cuyo desarrollo en el siglo XX ha sido relevante en el tratamiento e investigación de la corporeidad (LAZZARIS, 2020). La metodología seleccionada se compuso del enfoque biográfico narrativo (BOLIVAR, DOMINGO y FERNÁNDEZ, 2001) y la etnografía educativa (ROCKWELL, 2009), que permitió el acercamiento a los significados de las prácticas corporales en la enseñanza, poder percibirlos en el aula

4 Tesis doctoral: "Corporeidad y Buena Enseñanza en Profesores Memorables. Un estudio interpretativo en la Facultad de Humanidades de la UNMDP". Prof. Susana Lázaris. Director: Dr. Luis Porta. Universidad Nacional de Rosario. Argentina y entretejer sus sentidos en un conocimiento colectivo. Mediante entrevistas en profundidad a la docente, sus estudiantes y adscriptos, observaciones de clases, y el propio registro narrativo de la investigadora se fue comprendiendo la amalgama existente entre cuerpo, vida y enseñanza universitaria.

La expansión temática, epistémica y metodológica se observa claramente en esta última investigación del grupo. Primero porque ha podido abordar un aspecto sensible e íntimo como lo es el cuerpo y la noción de corporeidad luego de más de 10 años de trabajo con la misma docente memorable. La confianza, la rigurosidad y el compromiso ético del grupo para con ella permitió el despliegue de la expansión hacia cuestiones que se desarrollan en el aula universitaria pero que al mismo tiempo la trascienden. Segundo, esta investigación, nos llevó a un provocador desplazamiento instrumental en donde el registro fotográfico, fílmico, visual (AUGUSTOWSKY, 2007) e incluso la mirada etnográfica de las redes sociales de la profesora, nos permitió sumergirnos en la biografía más íntima de ella y poder interpretar, desde allí, la compleja urdimbre entre cuerpo, vida y enseñanza que emerge de sus clases y de los testimonios narrados por sus estudiantes, ayudantes y adscriptos.

En suma, en nuestro hábitat investigativo más próximo, la narrativa autobiográfica y su expansión se presenta posibilitando interceptar discursos con doble potencialidad: "la producción de conocimiento y el involucramiento del sujeto que narra" (GONZÁLEZ MONTEAGUDO, 2008, p. 40). En este contexto los soportes instrumentales y metodológicos desde los cuales sostenemos dicha expansión son sustanciales puesto que, mediante el resguardo de una ligazón coherente con los marcos epistemológicos y conceptuales, nos posibilitan alcanzar e interpretar los datos necesarios para la construcción de conocimiento en torno a la 
enseñanza, la pedagogía y la biografización de las vidas de quienes conforman los cotidianos educativos contemporáneos.

\section{Reflexiones Finales}

De "instrumento de recolección de datos" a "tecnologías de co-construcción de la experiencia", lo biográfico narrativo ha mutado inevitablemente al compás de los tiempos y las prácticas de investigación, afectándose progresivamente a medida que los marcos interpretantes de lo social, pero también de la micro esfera pedagógica, van transformándose.

Hablar de la "expansión" de lo biográfico-narrativo es ajustado a la historia-memoria aquí construida, aunque tal vez no sea suficiente para aludir a las resonancias que los procesos han gestado en estos años para la comunidad de investigadores de referencia.

Hace unos días, una colega me entrevistó a propósito de los 20 años de la asignatura Problemática Educativa, la materia del Ciclo de Formación Docente en la que me desempeño como Profesor Titular desde hace años. Coincidiamos en que es un espacio al que nos referimos como un organismo vivo. Al respecto le decía

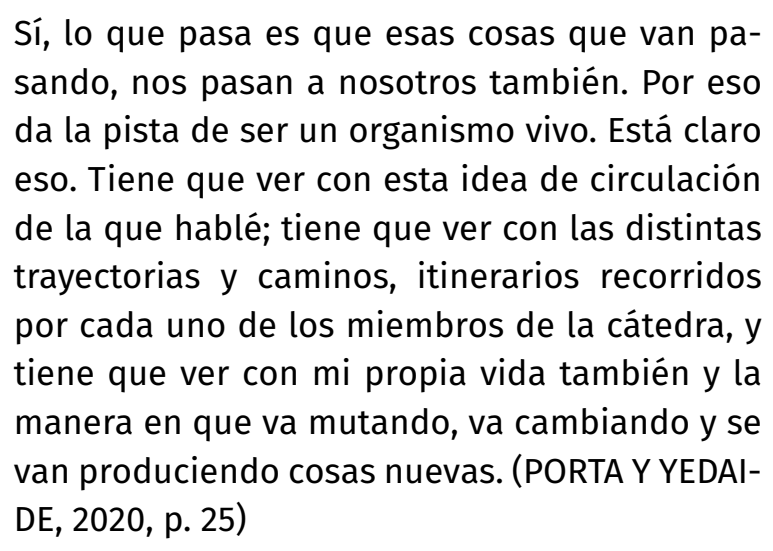

Precisamente por la ligazón con el proyecto vital, las tecnologías biográfico-narrativas no nos dejan indemnes. Incitan y reclaman la promesa del encuentro a partir de la intimidad-aquel ámbito de la vida que nos conec- ta con nuestra sensibilidad profunda, nuestra común vulnerabilidad y nuestros deseos-. La ciencia se vuelve humana en un sentido opuesto al de la más popular y nociva acepción del Humanismo (BRAIDOTTI, 2019). No nos permite ya anclarnos en el antropocentrismo despiadado, sino que nos transporta hacia una más humilde sustancia que compone y se compone con el todo. Lo narrativo, por el arrastre de lo singular e idiosincrático, no puede emanciparse de la cercanía. Y tal vez sea la proximidad, esa imposibilidad de la analgesia creada por la obstinación de la presencia de lo otro, aquello que nos salvará.

Con esta esperanza, y las expectativas que le nacen, lo biográfico auxilia a la investigación a no perder su corazón. No naufragará, sabemos hoy, en la sensiblería ni la indistinción con otras formas de producción del conocimiento. Investigar implica, fundamentalmente, sostener la pregunta. Esas preguntas a las que no podemos, como educadores, renunciar, porque interrogan sobre qué mundos construir y sostener, y qué lugar tienen las pedagogías en esos mundos. Y lo biográfico, aunque todavía implique andar por los bordes en este sentido ampliado que presentamos, completa con sentido y sentimiento la validez de estas preguntas.

\section{Referencias}

AGUIRRE, Jonathan, DE LAURENTIS, Claudia y BOXER, Matias. Lo (auto)etnográfico como territorio fecundo para una pedagogía queer: Narrativas de experiencias performáticas en el trayecto doctoral. Revista de Educación, v. 3. n. 18, p. 187-205, 2019. Disponible en: https://fh.mdp.edu.ar/revistas/index.php/r_educ/article/view/3756 Acceso en: 10 jul. 2020.

AGUIRRE, Jonathan. La formación docente en Argentina. Un estudio interpretativo de las políticas nacionales. El caso de los denominados Polos de Desarrollo (2000-2001). 2019. 656 f. Tesis de Docto- 
rado en Humanidades y Artes. Mención Educación. Facultad de Humanidades y Artes. Universidad Nacional de Rosario. Rosario. 2019.

ALVAREZ, Zelmira, PORTA, Luis y SARASA, Cristina. Una exploración del rol de los mentores en las trayectorias profesionales de los buenos docentes universitarios. Revista Práxis Educativa. v. 14, n. 14, p. 42-48. 2010. Disponible en: https://cerac.unlpam. edu.ar/index.php/praxis/article/view/431 Acceso en: 10 jul. 2020.

AHMED, Sara. Fenomenologia queer: orientaciones, objetos, otros. Trad. Javier Sáez del Álamo. Barcelona: Ediciones Bellaterra. 2019

AUGUSTOSVSKY, Gabriela. El registro fotográfico en la investigación educativa. En SVERDLICH, Ingrid comp. La investigación educativa, una herramienta de conocimiento y acción. Buenos Aires; Novedades Educativas. 2017. p. 251-278.

BRAIDOTTI, Rosi. A Theoretical Framework for the Critical Posthumanities. Theory, Culture y Society, v. 36, n. 6. 31-61. 2019. Disponible en: https: / journals. sagepub.com/doi/full/10.1177/0263276418771486 Acceso en: 10 jul. 2020.

BRANDA, Silvia. Enseñanzas que dejan huellas: la biografia escolar en las prácticas profesionales. Un estudio interpretativo en los estudiantes del Profesorado de Inglés de la Universidad Nacional de Mar del Plata. 2017, 354 fojas. Tesis de Doctorado en Humanidades y Artes. Mención Educación. Facultad de Humanidades y Artes. Universidad Nacional de Rosario. Rosario, 2017.

BOLIVIAR, Antonio. DOMINGO, José y FERNÁNDEZ, Miguel. La investigación biográfico-narrativa en educación. Enfoque y metodología. Madrid: La Muralla, 2001.

CANDELERO, Neldo. Ciencia, Arte, Medios Consideraciones en torno a la experiencia. En: Congreso nacional e internacional de Educación artística. "Hacia una Educación artística participativa, comprometida, e innovadora"; Primer Edición. 2016. Rosario. Actas de Congreso. Rosario. Ciudad Gótica, 2016. p. 5-52.

DE LAURENTIS, Claudia. La Formación del Profesorado: identidad profesional a partir de las narrativas biográficas de docentes. Un estudio interpretativo en el Ciclo de Formación Docente de los profesorados de la Facultad de Humanidades de la UNMDP. 2019. 420f. Tesis de Doctorado en Humanidades y Artes. Mención Educación. Facultad de Humanidades y Artes. Universidad Nacional de Rosario. Rosario, 2019.

DENZIN, Norman. Performance, hermeneutics, interpretation. En: UWE, Flick, The sage handbook of qualitative data collection. Londres: SAGE Publications Ltd., 2018. p. 200-216.

DENZIN, Norman y LINCOLN, Yvonna. El campo de la investigación cualitativa. Manual de investigación cualitativa. Vol. I. Barcelona: Gedisa, 2011.

DENZIN, Norman y LINCOLN, Yvonna. Manual de investigación cualitativa, IV: métodos de recolección y análisis de datos. México: Gedisa, 2015.

DI FRANCO, Graciela. El Campo de las Prácticas Profesionales en la formación del profesorado. Un estudio interpretativo en el Profesorado de Geografía de la Universidad Nacional de La Pampa. 2019. 364 f. Tesis de Doctorado en Humanidades y Artes. Mención Educación. Facultad de Humanidades y Artes. Universidad Nacional de Rosario. Rosario, 2019.

FINLEY. Susan. Investigación con base en las artes. En: DENZIN, Norman y LINCOLN, Yvonna Métodos de recolección y análisis de datos. Manual de investigación cualitativa. Vol. IV. Buenos Aires: Gedisa, 2015. p. 113-139

FLORES, Graciela. Dimensión ética de la enseñanza. Un estudio interpretativo de las prácticas de profesores universitarios memorables. 2018. 540f. Tesis de Doctorado Doctorado en Humanidades y Artes. Mención Educación. Facultad de Humanidades y Artes. Universidad Nacional de Rosario. Rosario, 2018.

FLORES, Valeria. Tropismos de la disidencia. Santiago de Chile: Palinodia, 2017.

FOUCAULT, Michael. La arqueología del saber. Buenos Aires: Siglo Veintiuno Editores. 2013 [1969].

FOUCAULT, Michael. Las palabras y las cosas. Una arqueología de las ciencias humanas. Buenos Aires: Editorial Siglo Veintiuno. 2011 [1966]. 
GERRARD, Jessica.; RUDOLPH, Sophie y SRIPRAKASH, Arathi. 'The Politics of Post-Qualitative Inquiry: History and Power'. Qualitative Inquiry, v. 23. n. 5, p. 384-394, 2017. Disponible en: https:/ / journals.sagepub.com/doi/abs/10.1177/1077800416672694 Acceso en: 10 jul. 2020.

GONZALEZ MONTEAGUDO, José. Historias de vida y teorías de la educación: tendiendo puentes. Cuestiones Pedagógicas, v. 19. n. 8, p. 207-232, 2008. Disponible en: https://dialnet.unirioja.es/servlet/articulo?codigo=4683179 Acceso en: 10 jul. 2020.

GUBER, Roxana. La etnografía. Método, campo y reflexividad. Buenos Aires: S. XXI. 2016.

HALBERSTAM, Jack. El arte queer del fracaso. Barcelona: Egales editorial, 2018.

HOLMAN Jones. Autoetnografía. En: DENZIN, Norman y LINCOLN, Ivonna. Métodos de recolección y análisis de datos. Manual de investigación cualitativa. Vol. IV. Buenos Aires: Gedisa, 2015. p. 262-315.

KEMMIS, Stephen. y MCTAGGART, Robin. La investigación acción participativa. La acción comunicativa y la esfera pública. En: DENZIN, Norman y LINCOLN Ivonna (comps.). Estrategias de investigación cualitativa. Manual de Investigación Cualitativa Vol. III. Barcelona: Gedisa, 2013. p. 361-439.

KUBY, Candece y CHRIST, Rebeca. Productive Aporias and Inten(t/s)ionalities of Paradigming: Spacetimematterings in an Introductory Qualitative Research Course. Qualitative Inquiry, v. 24, n. 4, p. 293-304, 2018. Disponible en: https:/ / journals.sagepub.com/ doi/abs/10.1177/1077800416684870 Acceso en: 10 jul. 2020.

LAKOFF, George y JOHNSON Mark. Metáforas de la vida cotidiana. Madrid: Colección Teorem, 1995.

LAZZARIS, Susana. Corporeidad y buena enseñanza en profesores memorables. Un estudio interpretativo en la Facultad de Humanidades de la UNMdP. 2020 305f. Tesis de Doctorado en Humanidades y Artes. Mención Educación. Facultad de Humanidades y Artes. Universidad Nacional de Rosario. Rosario, 2020.

LINCOLN, Yvonna. Los Comité de Conducta Ética y el Conservadurismo Metodológico. Los cuestionamientos del paradigma fenomenológico. El campo de la investigación cualitativa. En: DENZIN, Norman y LINCOLN, Ivonna. Manual de investigación cualitativa. Vol. I. Barcelona: Gedisa, 2011. p. 332-364.

LITWIN, Edith. El campo de la didáctica: en búsqueda de una nueva agenda. En: CAMILLONI, Alicia; DAVINI, Cristina; ELDESTEIN, Gloria; LITWIN, Edith; SOUTO, Marta y BARCO Susana. Corrientes didácticas contemporáneas. Buenos Aires: Paidós, 1996. p. 125-158.

LUPTON, Debora. Data selves: more-than-human perspectives. Oxford: Polity Press. 2019

MARTINEZ, Cristina. Enseñanza proyectual; vínculos entre comunidad de práctica y profesores memorables. Estudio interpretativo en el Taller de Diseño Arquitectónico “A” de la FAUD. UNMDP. 2017, $402 f$. Tesis de Doctorado en Humanidades y Artes. Mención Educación. Facultad de Humanidades y Artes. Universidad Nacional de Rosario. Rosario, 2017.

NORDSTROM, Susan. Antimethodology: Postqualitative Generative Conventions. Qualitative Inquiry v 24. n. 3 215-226. 2018. Disponible en https: / journals. sagepub.com/doi/abs/10.1177/1077800417704469?journalCode=qixa Acceso en: 10 jul. 2020.

RAMALLO, Francisco. El bachillerato como experiencia: Un abordaje biográfico narrativo a partir del Colegio Nacional de Mar del Plata, 1914-1940. 2017, 310f. Tesis de Doctorado en Humanidades y Artes. Mención Educación. Facultad de Humanidades y Artes Universidad Nacional de Rosario. Rosario, 2017.

ROCKWELL, Elsie. La experiencia etnográfica. Buenos Aires: Paidós, 2009.

PORTA, Luis, ÁLVAREZ, Zelmira, YEDAIDE, María Marta. Travesías del centro a las periferias de la formación docente. La investigación biográfico-narrativa y las aperturas a dimensiones otras del currículo. Revista Mexicana de Investigación Educativa, v. 19. N. 63, p. 1175-1193, Oct.-Dic. 2014. Disponible en http:// www.scielo.org.mx/pdf/rmie/ v19n63/v19n63a8.pdf Acceso en: 10 jul. 2020.

PORTA, Luis. Pedagogía doctoral y hábitat sensoriales. En: Simposio sobre Pedagogía Doctoral: In- 
vestigación, relatos y experiencias en la formación doctoral. Primera Edición. 2018. Mar del Plata. Actas de Simposio "Pedagogía Doctoral en Educación. Investigación, Relatos y Experiencias en la Formación Doctoral". Mar del Plata. Ed. Facultad de Humanidades- UNMdP. 2018. p. 1-5

PORTA, Luis y YEDAIDE, María Marta. Pedagogías vitales. Cartografias del pensamiento y gestos ético-políticos en perspectiva descolonial. Mar del Plata: EUDEM, 2017.

PORTA, Luis., AGUIRRE, Jonathan. y RAMALLO, Francisco. La expansión (auto)biográfica: Territorios habitados y sentidos desocultados en la investigación educativa. Revista Interritorios, v. 4, n. 7, p. 165-183, 2018. Disponible en https://periodicos.ufpe.br/revistas/interritorios/article/view/238248 Acceso en: 10 jul. 2020.

PORTA, Luis y AGUIRRE, Jonathan. La investigación (auto)biográfico-narrativa en la educación superior argentina. La expansión de horizontes metodológicos. En: AVENDAÑO, Fernando. Quehaceres de la investigación. Rosario: Homo Sapiens, 2020.

PORTA, Luis y YEDAIDE, María Marta. Pedagogías para hacer temblar mundos. Problemática Educativa como organismo vivo frente al deseo, la pregunta y la germinación de futuros. Revista de Educación, UNMDP. V. 21.2, p. 10-30, 2020. Disponible en: https://fh.mdp.edu.ar/revistas/index.php/r_educ/ article/view/4573/4603 Acceso en: 10 jul. 2020.

RIVERA CUSICANQUI, Silvia. Historias Debidas VIII: Silvia Rivera Cusicanqui. Canal Encuentro. 2017. Disponible en: https://www.youtube.com/watch?v=1q6HfhZUGhc Acceso en: 10 jul. 2020.

ROLNIK, Suely. Esferas de la insurrección. Apuntes para descolonizar el inconsciente. CABA: Tinta Limón, 2019.

SOUSA SANTOS, Boaventura. Crítica de la razón indolente: contra el desperdicio de la experiencia. Para un nuevo sentido común: la ciencia, el derecho y la política en la transición paradigmática. Vol. I. Bilbao: Desclée de Brouwer S.A, 2003.

SARASA, Cristina. Relatos entramados de futuras identidades profesionales docentes. Una indaga- ción narrativa en la formación inicial del Profesorado en Ingles. 2016, 349 f. Tesis de Doctorado en Humanidades y Artes. Mención Educación. Facultad de Humanidades y Artes. Universidad Nacional de Rosario. Rosario, 2016

SEDGWICH, Eve. Tocar la fibra: Afecto, pedagogía, performatividad. Madrid: Alpuerto, 2018.

SEGATO, Rita. La crítica de la colonialidad en ocho ensayos. Y una antropología por demanda. Buenos Aires: Prometeo Libros, 2015.

ST. PIERRE, Elizabeth. Haecceity: Laying Out a Plane for Post Qualitative Inquiry. Qualitative Inquiry, v. 23 n. 9, p. 686-698, 2017. Disponible en: https:/ /journals. sagepub.com/doi/abs/10.1177/1077800417727764 Acceso en: 10 jul. 2020.

ST. PIERRE, Elisabeth. The posts continue: Becoming. International Journal of Qualitative Studies in Education, v. 26, n. 1., p. 646-657, 2013. Disponible en: https://www.tandfonline.com/doi/abs/10.1080/095 18398.2013.788754 Acceso en: 10 jul. 2020.

ST. PIERRE, Elizabeth. A brief and personal history of post qualitative research: Toward "post inquiry". Journal of Curriculum Theorizing, v. 30 n. 2, p. 2-19, 2014. Disponible en: https://journal.jctonline. org/index.php/jct/article/view/521 Acceso en: 10 jul. 2020.

TAYLOR, S. Y BOGDAN, R. Introducción a los métodos cualitativos de investigación. Buenos Aires: Paidós, 2007.

WAYAR, Marlene. Palabras de fuego: Teoría travesti-trans sudamericana. Travesti/ Una teoría lo suficientemente buena. Buenos Aires: Muchas Nueces, 2008.

TRUEBA, Sebastian. Profesores memorables para los docentes formadores de los profesorados de Educación Física de la ciudad de Mar del Plata. 2019, 268f. Tesis de Doctorado en Humanidades y Artes. Mención Ciencias de la Educación. Universidad Nacional Rosario. Rosario, 2019.

YEDAIDE, María Marta. El relato "oficial" y los "otros" relatos sobre la enseñanza en la formación del Profesorado: un estudio interpretativo en la Facultad 
de Humanidades, UNMdP. 2016, 351f. Tesis de Doctorado en Humanidades y Artes. Mención Educación.
Facultad de Humanidades y Artes. Universidad Nacional de Rosario. Rosario, 2016.

Recebido em: 29/09/2020

Aprovado em: $14 / 12 / 2020$

Luis Porta é Doctor en Filosofía y Ciencias de la Educación por la Universidad de Granada, España. Docente e Investigador categoría 1, Departamento de Ciencias de la Educación. Facultad de Humanidades (UNMdP) e Investigador Principal de CONICET. Profesor Titular Regular de la Cátedra Problemática Educativa y de Sociología de la Educación. Director del Centro de Investigaciones Multidisciplinarias en Educación (CIMed-UNMdP). Coordinador del Programa de Doctorado en Investigación Narrativa, Biográfica y AutoBiográfica en Educación de la Universidad Nacional de Rosario, Argentina. E-mail: luisporta510@gmail.com 